\title{
A Novel Visualization Tool for Evaluating Medication Side-Effects in Multi-drug Regimens
}

\author{
Jon Duke ${ }^{1,2}$, Anthony Faiola ${ }^{1}$, and Hadi Kharrazi ${ }^{1}$ \\ ${ }^{1}$ Indiana University Purdue University Indianapolis, School of Informatics \\ 535 W. Michigan St., Indianapolis, Indiana 46202 \\ ${ }^{2}$ Regenstrief Institute, Medical Informatics, HITS 2000 \\ 550 W. 10th Street Indianapolis, IN 46209 \\ jduke@regenstrief.org, afaiola@iupui.edu, kharrazi@iupui.edu
}

\begin{abstract}
The evaluation and management of medication side-effects is a common and complex task for physicians. Information visualization has the potential to increase the efficiency and reduce the cognitive load involved in this process. We describe the design and development of Rxplore, a novel tool for assessing medication side-effects. Rxplore supports simultaneous lookup of multiple medications and an intuitive visual representation of query results. In a pilot study of Rxplore's usability and utility, physicians rated the system highly for efficiency, intuitiveness, and clinical value.
\end{abstract}

Keywords: information visualization, medical informatics, adverse reactions, medications, side-effects.

\section{Introduction}

Medication side-effects are a significant cause of patient morbidity and mortality in the United States, responsible for over 100,000 deaths [1] and an estimated cost of $\$ 77$ billion annually [2]. While physicians are generally aware of the most common and serious side-effects of the medications they prescribe, it is extremely difficult for them to memorize all the potential effects of a given drug. Indeed, Food and Drug Administration (FDA) labels typically list dozens, if not hundreds, of reported reactions for a single agent [3].

When a patient is taking multiple medications, the doctor's decision making process becomes even more complex. Not only does the sheer number of possible sideeffects grow, but the effects themselves may overlap synergistically and increase the patient's risk of an adverse event [4]. Studies on polypharmacy - the use of multiple or excess prescription drugs - show that half of Americans over 65 take at least five medications regularly and these numbers are continuing to rise [5]. Therefore evaluating a patient's drug regimen for potential adverse reactions has become an increasingly difficult task.

A variety of electronic drug information resources have been created to support the physician in this process. These resources typically list the side-effects associated

J.A. Jacko (Ed.): Human-Computer Interaction, Part IV, HCII 2009, LNCS 5613, pp. 478-487, 2009. Springer-Verlag Berlin Heidelberg 2009

Duke, J., Faiola, A., \& Kharrazi, H. (2009). A novel visualization tool for evaluating medication sideeffects in multi-drug regimens. In Human-Computer Interaction. Interacting in Various Application Domains (pp. 478-487). Springer Berlin Heidelberg. http://dx.doi.org/10.1007/978-3-642-02583-9_52 
with each medication and note how frequently these effects are reported in clinical trials. Yet even the most efficient systems require physicians to look up medications one-by-one. Furthermore, physicians must rely on memory or note-taking to organize the information they uncover while interacting with these systems. In this paper, we describe the development of a new visualization tool designed to address these issues and to expedite the review of side-effect data. We also present the results of a pilot study on the usability and clinical utility of this system.

\section{Theory and Background: Information Visualization and Biomedical Informatics}

Information visualization is a mapping technique that utilizes visual graphics to represent non-spatial abstract datasets in physical space [6]. Visualization enables users to make discoveries and decisions regarding complex data, elucidating subtle patterns and making information-intensive tasks more manageable. In the following section, we explore the theoretical underpinnings of information visualization as a means to augment human cognition. Then we review some current applications of visualization in the healthcare setting.

\subsection{Information Visualization and Augmented Cognition}

A prime challenge of modeling a meaningful visualization is identifying an appropriate visual mapping of the information that best supports correlations and relationships between the unstructured data; thereby enhancing new understanding. How people perceive, understand, and apply information visualization is essential to grasp the relationship between human-computer interaction and human cognition. As Heer et al. state, information visualization seeks to "augment human cognition by leveraging human visual capabilities to make sense of large collections of abstract information" [7].

The amplification of cognition through information visualization modeling, joined with data analysis, provides users interaction with a support for more sense-making of large and complex datasets. For example, amplifying cognition through information visualization both increases cognitive resources by expanding working memory and reduces search by representing large datasets in a small space. As Beale et al. state [8], cognitive amplification through the use of visualization methods "helps to shift the work load from the cognitive to the perceptual system; expands the working memory; and allows a high level of interaction... [thus aiding in the users'] confirmation and discovery of knowledge". Furthermore, information visualization supports relationships of perceptual inference that would otherwise be quite difficult to reproduce.

One of the greatest benefits of information visualization is that it (unlike static media) provides a means to manipulate and enable the exploration of a space of parameter values $[8,9]$. This is observed in the theory of external cognition as applied to design issues and an analysis of how graphical representations are used during cognitive activities such as problem-solving [10]. Specifically, external cognition refers to the "interaction between internal and external representations when performing cognitive tasks such as learning" [11]. The design dimensions of information visualizations are intended to help researchers and practitioners, through 
combinations of visualizations, to experience a type of "computational offloading, the extent to which different external representations vary the amount of cognitive effort required to carry out different activities" [11].

This concurs with Norman's early work $[12,13]$ in the psychology of humancomputer interaction, in which he argued for a fundamental paradigm shift in understanding the way interactive products are designed. For example, he suggested that well-designed artifacts should reduce the need for users to remember large amounts of information. In the section titled "The Conspiracy against Memory" [12], Norman highlights our inability to freshly retain many items, i.e., the way these items work and the way they relate to one another. He asserts that the human mind is limited in its ability to think deeply about any given topic, primarily because of the restricted capacity of working memory. For this reason, visual aids, such as information visualization, are necessary to support both cognition and an array of learning processes, as well as to reduce human error. In sum, researchers and system designers need to find ways to arrange complex systems that are visible to reduce information in memory and reflect human logic.

Consequently, we can consider the visualization interface functions as the contact point for interaction, where information enters through the human sensory system, is organized, and finally recognized to ensure an error-free display of the information. When interacting with a visualization system, users may process the information into different forms, e.g., perception and transformation of understanding. On one hand, the computational modeling of data visualization is critical for an accurate display of information. On the other, interaction design and information design are at the heart of information visualization. Most importantly, empirical findings have shown that properly designed visualizations have the potential to give a clear picture at a glance. This is because the laws of semantic configuration must be learned, but images provide automatic comparisons and obvious relationships [14].

\subsection{Information Visualization in Healthcare}

Information retrieval is an important part of the daily routine of physicians and other healthcare providers. Yet the body of knowledge in medicine is increasing enormously, forcing clinicians to rely on various external sources of medical information [15]. These resources can range from textbooks, to external websites, or even to fully integrated clinical decision support (CDS) systems. When discussing these tools, it is important to differentiate between those designed purely for "data availability" and those which have extended this concept to include the notion of "information extraction" [16]. Resources that offer data availability alone (such as a textbook or patient chart) will provide the physician with accurate information but leave him with the burden of collecting, maintaining in working memory, and interpreting these data. Conversely, resources that move beyond availability and provide assistance with information extraction (such as context-sensitive clinical reminders or real-time cardiac telemetry) can reduce the physician's cognitive load. Information visualization has been shown to be one such successful method of supporting extraction and reducing cognitive load in the medical setting $[17,18]$.

There are currently a number of visualization applications in clinical use. One such application that is often overlooked is the electrocardiogram (EKG), which is 
essentially a visual representation of the heart's direction and force throughout the cardiac cycle. More traditional forms of data visualization would be graphical monitoring of blood pressure, pulse, and other vital signs in the intensive care unit [19]. Graphical displays of laboratory results have also become a standard feature of electronic medical record systems in both the hospital and outpatient settings. More recently, work has been done to visually represent patient clinical histories, such as diagnoses, medications, surgeries, and so forth [20,21]. These clinical visualizations provide an integrated view of the temporal characteristics of a patient's history, giving the physician an integrated high-level view of the patient's symptoms and treatments over time.

Despite these areas of progress, there are relatively few reports in the literature of visualization techniques being applied to the evaluation of medications and their sideeffects. One such application is DOPAMINE, a tool for visualizing drug therapeutic classes, but this work was primarily focused on ontology development rather than clinical practice [22]. Another application is called "Mister VMC," a tool which implements an anatomic representation technique to portray side-effects, contraindications, and interactions in a real-time clinical context. A study showed improved accuracy and speed of obtaining drug information using this tool when compared with a standard text-based system [23]. However the major drawback of this system is its complex iconography, requiring prior training in a proprietary visual language to use the application effectively.

\subsection{Adverse Drug Reactions and Clinical Decision Support}

While the availability of graphical decision support systems for drug information is quite limited, text-based decision support applications have been increasingly adopted by physicians. Two prime examples are Epocrates and UpToDate. These applications have been shown to be effective in reducing prescribing errors and assisting clinical decision-making $[24,25]$. Both solutions are popular, with 100,000 Epocrates users and over 300,000 UpToDate users visiting their websites every month [26,27].

Whether accessing the PDA or Desktop version, a user interacts with these applications by searching for a medication of interest and having the option to choose from several pertinent categories of prescribing information. Side-effect data are generally displayed in a slightly condensed (UpToDate) or significantly condensed (Epocrates) form. Users typically write down or just remember the information they need before closing the application and returning to the task at hand. There is no current method of looking up multiple medications simultaneously, or of searching across a set of medications for a particular side-effect.

While Epocrates and UpToDate are both excellent information resources and have improved the efficiency of retrieving drug side-effect data, they have not significantly reduced the overall cognitive complexity of the process. In other words, their focus remains on data availability rather than information extraction. In the following section, we describe the creation of a novel drug information resource which aims to support both these elements in equal measure through effective data visualization methods. 


\section{Methods}

\subsection{Development of the Rxplore Visualization Tool}

The development goals were to create a tool which would allow users to rapidly retrieve side-effect data on multiple medications and to deliver these results in an easily interpretable manner with minimal cognitive load. The development process comprised two stages: 1) creation of a database containing quantitative data on medication side-effects 2) construction of a web interface to query this database and display the results of multiple queries in graphical form.

Creation of the Quantitative Adverse Reaction Knowledgebase (QuARK). Adverse reaction data were extracted from $250 \mathrm{FDA}$-approved medication labels by both manual and natural language processing extraction methods. These data were based on multiple sources (e.g., clinical trials, post-marking reports) and could be either quantitative or qualitative. In order to account for these differences, we constructed algorithms to simplify the relationship of each medication and side-effect to a singular value called the Rxplore Score (Table 1).

Table 1. Example algorithms used in generating Rxplore Scores

\begin{tabular}{|c|c|c|c|}
\hline Reporting Format & Example & Algorithm & Rxplore Score \\
\hline Drug and Placebo & $\begin{array}{l}\text { Nausea seen in } 27 \% \text { on Drug } \\
\mathrm{X} \text { and } 12 \% \text { placebo }\end{array}$ & Drug $\%$ - Placebo \% & $27-12=15$ \\
\hline Frequency Range & $\begin{array}{l}\text { Itching occurred in between } \\
3 \% \text { and } 9 \% \text { of patients }\end{array}$ & $\mathrm{LB}+(\mathrm{UB}-\mathrm{LB}) / 3$ & $3+(9-6) / 3=5$ \\
\hline Qualitative Terms & Rash occurred "rarely" & $\begin{array}{l}\text { Expert consensus }+ \\
\text { Matching terms in } \\
\text { quantitative datasets }\end{array}$ & 0.3 \\
\hline
\end{tabular}

This scoring system was not designed to be an exact measurement of frequency, but rather to provide a conceptual gauge reflecting the association of each drug with a particular side-effect. The original data were retained in the knowledge-base to allow recalculation of the scores in case of an algorithm modification. The final Rxplore database contains information on over 16,000 medication and side-effect pairs.

Interface and Visualization Design. A web-based interface to the Rxplore data was then developed using ASP.NET, Flash, and SQL Server. The requisite features were: 1) the ability to create a list of multiple medications 2) a means to search for a specific side-effect across this entire list and 3) an intuitive visual display of the search results. For the visualization, we selected a stacked-bar format in which each row represents a single side-effect, and each bar within the row reflects the Score of that effect for a given medication (Figure 1). Bars are differentiated by color to allow the user to see which medications are associated with a particular effect. The size of each bar is proportional to the calculated Rxplore Score, so that when seen as a collective, the graph conveys instantaneously which medications are most likely to cause a given effect. 


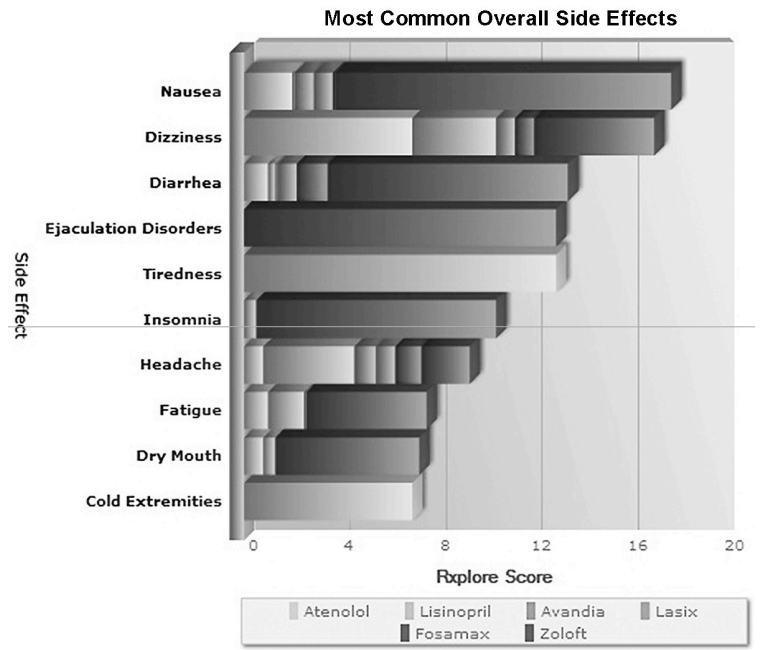

Fig. 1. A summary graph from Rxplore, depicting the most common side-effects reported with a regimen consisting of six different medications. Larger bars suggest a higher associated frequency between the drug and a side-effect.

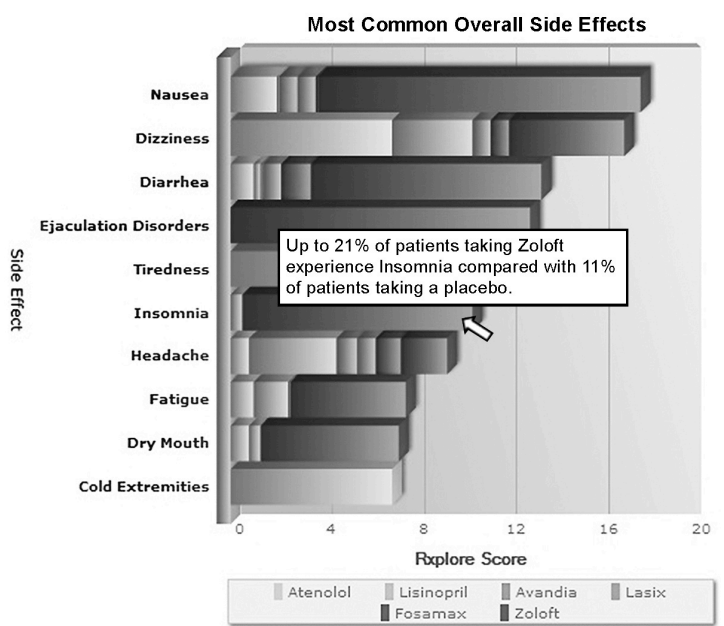

Fig. 2. A summary graph of medication side-effects with detailed frequency data displayed. Moving the mouse over a bar on the graph retrieves the specifics for that medication and sideeffect combination.

An "overview plus detail" model was used to provide textual side-effect data on demand. For example, if the user wants to retrieve the details on the relationship between Zoloft and Insomnia, he can hover over the graph in the Insomnia row on the Zoloft bar and the desired information will be displayed (Figure 2). Hence, the visualization technique allows for rapid interpretation of the high-level data as well as expedient retrieval of lower level details. 
In addition to displaying the most common side-effects (Figure 2) for a medication regimen, the user can also retrieve the most common effects of a particular type (e.g. cardiac, gastrointestinal) or search for an individual side-effect of interest. Medication regimens and custom side-effects lists can be stored for future review and adjustment.

\subsection{Evaluation Study}

Research Questions. In conducting a preliminary evaluation of Rxplore, we sought to answer two basic questions. First, how do health care providers rate the system's usability? Specifically we were interested in its learnability, efficiency, and ease of use. Second, how do these providers rate the system's clinical utility? Our metrics for utility were perceived clinical value and relevance to doctors' daily work.

Participants. We invited 63 physicians and nurses at the Indiana University Medical Group to participate in this evaluation study, of whom 10 enrolled.

Treatment. The subjects were first shown an introductory video to explain the concept and features of the Rxplore tool. Then, they were presented with two patient scenarios, each containing a clinical description, current medication use, and recent history and physical exam findings. For each scenario, there were 5 questions regarding the patient's symptoms and potential causal relationship of the patient's medications (e.g. "Which of these medications is most likely to be causing his Nausea?"). Subjects worked through the questions independently using the Rxplore tool. Upon completion of the tasks, they were given an online questionnaire regarding the system's usability and clinical utility. Follow-up interviews were conducted to gain further insight into the subject's experiences with Rxplore.

\section{Results}

\subsection{Demographics}

Participants consisted of $90 \%$ physicians while $10 \%$ were with nursing backgrounds. The subjects described themselves as $56 \%$ avid users of computers, $33 \%$ average users and $11 \%$ with poor computer skills. We explored their current drug information needs and found that $67 \%$ used an electronic resource to look up medication sideeffects on a daily basis, $22 \%$ did so a few times per week, and $11 \%$ accessed such a resource only a few times a month.

\subsection{Utility}

Rxplore's effectiveness and clinical utility were measured by task-oriented selfreported questions. For example, the users were asked to rate their experience when performing tasks such as adding a medication, deleting a medication, finding a specific side-effect or selecting a side-effect category. Rxplore was rated by average $94.5 \%$ effective in accomplishing the aforementioned tasks. 


\subsection{Usability}

A number of questions focused on the usability of the system. Its Intuitiveness rated 4.7 out of 5 while Ease to Use rated 4.6 out of 5. Participants overall found the system appealing (4.6 out of 5) and also aesthetically pleasing (4.9 out of 5). Subjects rated Rxplore's Speed and Time-Saving potential as 4.4 out of 5. Furthermore, when asked if it was faster than their current source of drug information, subjects rated Rxplore at $96 \%$ for retrieving all common side-effects and $100 \%$ for retrieving the frequency of a specific side-effect.

Participants were also asked specific questions about the system's visual concept and the meaning of its elements. For example, regarding the clarity of the bars in predicting the risks of experiencing a side-effect, users rated the system $100 \%$. The same result was concluded regarding the relationship between multiple bars on the same side-effect and several medications causing the effects. One area of weakness was in recognizing Rxplore as a conceptual measure rather than as a precise statistical metric. This concept received only a $60 \%$ rating for clarity.

\subsection{Usefulness}

Regarding the potential clinical value, subjects rated the system 4.5 out of 5 in terms of relevancy to their work and 4.9 out of 5 in terms of usability in clinical practice (Figure 8). Overall satisfaction was high, with $90 \%$ of subjects rating their experience with Rxplore as Excellent or Good; none had a Fair or Poor experience. Likelihood of using Rxplore again was high (4.6 out of 5) as was the willingness to recommend Rxplore to colleagues (4.6 out of 5 ).

\section{Discussion}

The benefit of visualizing complex information has been established in multiple health care settings. In this paper, we have described a new use of information visualization for the purposes of evaluating medication side-effects. This visualization tool, known as Rxplore, has two main advantages over current methods of researching adverse drug reactions. First, it allows for retrieval of information on multiple medications simultaneously. Second, by quantifying the relationship between each medication and its associated effects, Rxplore can provide an immediately interpretable visual representation of this complex set of data. Although small in size, our pilot study's cohort of physicians demonstrated considerable enthusiasm for the tool's usability and potential value in the clinical setting.

By using standard visualization methods, including stacked bar graphs and rollover text boxes for detailed data, Rxplore's learning curve was kept to a minimum. No dedicated training was required to use the system, and participants rated it highly in terms of both ease of use and intuitiveness. While quantitative measures of cognitive load were not recorded in this study, subjects reported the system was efficient, timesaving, and faster than their current drug information resource. This increased efficiency may relate to decreased demands on working memory and the computational off-loading achieved by providing information on multiple medications in a single image. Typically, users would need to remember their findings for each individual 
drug as the data are looked up sequentially. With Rxplore's simultaneous graphical retrieval technique, cognitive load previously spent on memory tasks can be shifted to the interpretation of the data. This shift in effort results in improved information extraction without additional work on the part of the physician.

Despite its potential benefits, Rxplore does have several limitations as well. The most significant issue being the reliability of the underlying algorithms used to calculate the Rxplore Scores. Though validation studies are underway, these Scores are necessarily coarse reflections of the complex (and sometimes conflicting) data available regarding medication side-effect frequencies. In absence of a gold standard, physicians must rely on the body of available knowledge and their own clinical experience to judge potential adverse reactions in any given patient. Thus, the Rxplore Scores have been designed not as a precise statistical model of side-effect likelihood, but rather as a guide to encourage physicians to explore the underlying data in a more efficient manner.

The challenge in any information visualization is to ensure that the viewer understands the full meaning of the displayed information. With Rxplore, avoiding hasty assumptions and encouraging exploration of the underlying data is absolutely essential. Based on the study results, several physicians did not fully understand the use of Rxplore Score as a conceptual rather than statistical metric. Thus, we must present the methodology to users more clearly to ensure clinical judgments are based on the full spectrum of available data.

The major limitation of our pilot study was its small size and absence of a control resource for comparison. Upcoming work will include a comparative study between Rxplore and traditional drug information resources. We will also expand the number of participants as well as the acquisition of quantitative data for measuring usability, efficiency, and cognitive load. Finally, integration of Rxplore into an existing electronic medical record system is now underway and will provide a rich test bed for further investigation into the system's use.

\section{References}

1. Lazarou, J., Pomeranz, B.H., Corey, P.N.: Incidence of Adverse Drug Reactions in Hospitalized Patients: A Meta-analysis of Prospective Studies. JAMA 279, 1200-1205 (1998)

2. Ernst, F.R., Grizzle, A.J.: Drug-related morbidity and mortality: updating the cost-ofillness model. J. Am. Pharm. Assoc. 41, 192-199 (2001)

3. DailyMed: About DailyMed, http://dailymed.nlm.nih.gov/dailymed/about.cfm

4. Veehof, L.J.G., et al.: Adverse drug reactions and polypharmacy in the elderly in general practice. European Journal of Clinical Pharmacology 55, 533-536 (1999)

5. Kaufman, D.W., et al.: Recent Patterns of Medication Use in the Ambulatory Adult Population of the United States: The Slone Survey. JAMA 287, 337-344 (2002)

6. Voigt, R.: An Extended Scatterplot Matrix and Case Studies in Information Visualization. Published as Diplomarbeit (2002),

http: //www.vrvis.at/via//resources/DA-RVoigt/DA.pdf

7. Heer, J., Card, S., Landay, J.P.: A Toolkit for Interactive Information Visualization. In: Proceeding of the ACM Conference on Human Factors in Computing Systems, pp. 421430 (2005) 
8. Thomas, J.J., Cook, K.A.: Illuminating the Path. 200 (2005)

9. Card, S.K., Mackinlay, J.D., Shneiderman, B.: Readings in Information Visualization 686 (1999)

10. Scaife, M., et al.: External Cognition: How Do Graphical Representations Work. International Journal of Human-Computer Studies 45, 185-213 (1996)

11. Rogers, Y.: New theoretical approaches for human-computer interaction. Annual Review of Information Science and Technology 38, 87-143 (2004)

12. Norman, D.A.: The Design of Everyday Things 257 (1990)

13. Norman, D.A., Draper, S.W.: User Centered System Design 526 (1986)

14. Gregg, L.W., Bower, G.H., University, C.: Cognition in Learning and Memory, pp. 51-88 (1972)

15. Holzinger, A.: HCI and Usability for Medicine and Health Care 458 (2007)

16. Workman, M., Lesser, M.F., Kim, J.: An Exploratory Study of Cognitive Load in Diagnosing Patient Conditions. Int. J. Qual. Health Care 19, 127-133 (2007)

17. Shachak, A., et al.: Primary Care Physicians' Use of an Electronic Medical Record System: A Cognitive Task Analysis. J. Gen. Intern. Med. (2009)

18. Stoicu-Tivadar, L., Stoicu-Tivadar, V.: Human-Computer Interaction Reflected in the Design of User Interfaces for General Practitioners. International Journal of Medical Informatics 75, 335-342 (2006)

19. Wenkebach, U., Pollwein, B., Finsterer, U.: Visualization of Large Datasets in Intensive Care. In: Proc. Annu. Symp. Comput. Appl. Med. Care, pp. 18-22 (1992)

20. Shahar, Y., Cheng, C.: Intelligent visualization and exploration of time-oriented clinical data. Top Health Inf Manage 20, 15-31 (1999)

21. Plaisant, C., Mushlin, R., Snyder, A., Li, J., Heller, D., Shneiderman, B.: LifeLines: Using Visualization to Enhance Navigation and Analysis of Patient Records. In: Proc. AMIA Symp., pp. 76-80 (1998)

22. Wroe, C.J., Solomon, W.D., Rector, A.L., Rogers, J.: Dopamine: A Tool for Visualizing Clinical Properties of Generic Drugs. In: Lavrac, B.K.N., Miksch, S. (eds.) International Workshop on Intelligent Data Analysis in Medicine and Pharmacology. The Fifth Workshop on Intelligent Data Analysis in Medicine and Pharmacology (2000)

23. Lamy, J., Venot, A., Bar-Hen, A., Ouvrard, P., Duclos, C.: Design of a Graphical and Interactive Interface for Facilitating Access to Drug Contraindications, Cautions for Use, Interactions and Adverse Effects. BMC Medical Informatics and Decision Making 8, 21 (2008)

24. Berner, E.S., et al.: Improving Ambulatory Prescribing Safety with a Handheld Decision Support System: A Randomized Controlled Trial. J. Am. Med. Inform. Assoc. 13, 171179 (2006)

25. Wilcox, R.A., Whitham, E.M.: Reduction of Medical Error at the Point-of-Care Using Electronic Clinical Information Delivery. Internal Medicine Journal 33, 537-540 (2003)

26. Quantcast UpToDate Profile, http: //www. quantcast.com/uptodate.com

27. Quantcast Epocrates Profile, http://www.quantcast.com/epocrates.com 